\title{
REMARKS ON DIMENSION OF HOMOLOGY SPHERES WITH ODD NUMBERS OF FIXED POINTS OF FINITE GROUP ACTIONS
}

\author{
Shunsuke TAMURA \\ (Received 12 May 2019 and revised 15 August 2019)
}

\begin{abstract}
For each positive integer $m$, an arbitrary finite non-solvable group acts smoothly on infinitely many standard spheres with exactly $m$ fixed points. However, for a given finite non-solvable group $G$ and a given positive integer $m$, all standard spheres do not admit smooth actions of $G$ with exactly $m$ fixed points. In this paper, for each of the alternating group $A_{6}$ on six letters, the symmetric group $S_{6}$ on six letters, the projective general linear group PGL $(2,9)$ of order 720 , the Mathieu group $M_{10}$ of order 720 , the automorphism group $\operatorname{Aut}\left(A_{6}\right)$ of $A_{6}$ and the special linear group SL $(2,9)$ of order 720 , we will give the dimensions of homology spheres whose fixed point sets of smooth actions of the group do not consist of odd numbers of points.
\end{abstract}

\section{Introduction}

Let $G$ be a finite group and $M$ a smooth manifold. A smooth action of $G$ on $M$ is a group homomorphism $\Phi$ from $G$ to the diffeomorphism group of $M$. Throughout this paper, all manifolds and all actions of finite groups on manifolds will be assumed smooth. For a manifold $M$ with $G$-action and a subgroup $H$ of $G$, let $M^{H}$ denote the $H$-fixed point set of $M$ : the set of all points $x \in M$ such that $\Phi(h)(x)=x$ for all $h \in H$. The $H$-fixed point set $M^{H}$ is a submanifold of $M$ with $N_{G}(H) / H$-action, where $N_{G}(H)$ is the normalizer of $H$ in $G$. An action of $G$ on $M$ is called a one-fixed-point action if $M^{G}$ consists of exactly one point. More generally, if $M^{G}$ consists of an odd number of points, we call such an action $G$ on $M$ an odd-fixed-point action.

For a commutative ring $R$ with unity, an $R$-homology sphere is a closed manifold having the same homology groups with coefficients in $R$ as a sphere. In this paper, a $\mathbb{Z}$-homology sphere will be simply called a homology sphere. A finite group $G$ is called a mod-P hyperelementary group if there exists a normal sequence $P \unlhd H \unlhd G$ such that $P$ and $G / H$ are of prime power order, and $H / P$ is cyclic. It is easy to see that spheres do not admit one-fixedpoint actions of mod- $\mathcal{P}$ hyper-elementary groups. The converse was proven by Laitinen and Morimoto [10]: Every finite Oliver group $G$, i.e. $G$ is not a mod- $\mathcal{P}$ hyper-elementary group, acts on some spheres with exactly one fixed point. Thus, the answer to the determination of finite groups acting on spheres with exactly one fixed point is already known. Furthermore,

2010 Mathematics Subject Classification: Primary 57S25; Secondary 55M35.

Keywords: finite group action; fixed point; tangential representation.

(C) 2020 Faculty of Mathematics, Kyushu University 
the problem of the dimension of spheres admitting one-fixed-point actions of finite groups has also been completed. Furuta [9], Demichelis [8] and Buchdahl, Kwasik and Schultz [6] showed that, if there exists a one-fixed-point action on a finite group on a sphere $S$, then the dimension of $S$ is greater than or equal to six. Conversely, the $n$-dimensional sphere $S^{n}, n \geq 6$, has one-fixed-point actions of the alternating group $A_{5}$, which was proven by Morimoto [11-13] for $n=6$ and $n \geq 9$, and by Bak and Morimoto $[1,2]$ for $n=7,8$.

In addition to the above results, we will describe some results on one- or odd-fixed-point actions of finite groups on spheres. Stein [19] constructed an effective one-fixed-point action of the special linear group $\operatorname{SL}(2,5)$ on $S^{7}$. On the other hand, Borowiecka [3] proved that $\operatorname{SL}(2,5)$ does not act effectively on any eight-dimensional homology sphere with exactly one fixed point. Recently, Borowiecka and Mizerka [4] gave many pairs $(G, S)$, where $G$ is a finite Oliver group with $|G| \leq 216$ and $S$ is a sphere with $\operatorname{dim} S \leq 10$, such that $G$ does not act on $S$ with exactly one fixed point, and Morimoto and Tamura [15] showed that, if there exists an effective odd-fixed-point action of the symmetric group $S_{5}$ (respectively $\operatorname{SL}(2,5)$ ) on a $\mathbb{Z}$ (respectively $\mathbb{Z}_{2}$ )-homology sphere $\Sigma$, then $\operatorname{dim} \Sigma$ does not lie in $\{0,1,2,3,4,5,7,8,9,13\}$ (respectively $\{0,1,2,3,4,5,6,8,9\}$ ). In this paper, we denote by $[a . . b]$ the set of integers $m$ such that $a \leq m \leq b$, by $\mathbb{N}$ the set of positive integers and by $\mathbb{Z}_{\geq 0}$ the set of non-negative integers, i.e. $\mathbb{Z}_{\geq 0}=\mathbb{N} \cup\{0\}$. Our main results are the following.

THEOREM 1.1. Let $\Sigma$ be a $\mathbb{Z}_{2}$-homology sphere. There are no effective odd-fixed-point actions of $A_{6}$ and $\operatorname{SL}(2,9)$ on $\Sigma$ if the dimension of $\Sigma$ lies in

$$
T_{A_{6}}=[0 . .7] \cup[9 . .12] \cup\{14,15\} \cup\{19,20\}
$$

and

$$
T_{\mathrm{SL}(2,9)}=[0 . .15] \cup[17 . .20] \cup\{22,23\} \cup\{27\},
$$

respectively.

THEOREM 1.2. Let $\Sigma$ be a homology sphere. There are no odd-fixed-point actions of $S_{6}$, $\operatorname{PGL}(2,9), M_{10}$ and $\operatorname{Aut}\left(A_{6}\right)$ on $\Sigma$ if the dimension of $\Sigma$ lies in

$$
\begin{aligned}
T_{S_{6}} & =[0 . .15] \cup[17 . .20] \cup[22 . .24] \cup[27 . .29] \cup\{33\} \cup\{38\}, \\
T_{\mathrm{PGL}(2,9)} & =[0 . .7] \cup[9 . .15] \cup[19 . .23] \cup[29 . .31] \cup\{39\}, \\
T_{M_{10}} & =[0 . .15] \cup[17 . .24] \cup[27 . .31] \cup\{33\} \cup[37 . .40] \cup\{47\} \cup\{49\}
\end{aligned}
$$

and

$$
T_{\text {Aut }\left(A_{6}\right)}=[0 . .15] \cup[17 . .24] \cup[27 . .31] \cup\{33\} \cup[37 . .40] \cup\{47\} \cup\{49\},
$$

respectively.

Here, $A_{6}$ and $S_{6}$ denote the alternating and the symmetric groups on six letters, respectively, and $\operatorname{SL}(2,9)$ and $\operatorname{PGL}(2,9)$ denote the special linear and the projective general linear groups of degree two over the finite field $\mathbb{F}_{9}$ with nine elements, respectively. And $M_{10}$ denotes the Mathieu group of degree 10: the definition in this paper is the subgroup of the symmetric group $S_{10}$ defined by

$$
\left\langle g, h \in S_{10} \mid g^{2}=h^{8}=\left(g h^{4}\right)^{3}=g h g h g h g h^{-2} g h^{3} g h^{-2}=e\right\rangle,
$$


where $g=(2,3)(4,6)(5,7)(8,9), h=(1,2)(3,4,7,9,10,8,6,5)$, and $e$ is the identity element in $S_{10}$. Finally, Aut $\left(A_{6}\right)$ denotes the automorphism group of $A_{6}$.

Theorem 1.1 is obtained from Theorem 3.4 for $A_{6}$, and from Theorem 3.6 for $\operatorname{SL}(2,9)$, and Theorem 1.2 follows from Theorem 4.3.

\section{Preliminaries for proofs of Theorem 1.1 and Theorem 1.2}

The following three facts are useful for our study.

Proposition 2.1. (cf. [5, Ch. III, Theorem 4.3]) Let $p$ be a prime number, $G$ a finite group of p-power order and $X$ a compact manifold with $G$-action. Then the Euler characteristic $\chi\left(X^{G}\right)$ is congruent modulo $p$ to the Euler characteristic $\chi(X)$.

LEMMA 2.2. (Smith's theorem [18], cf. [5, Ch. III, Theorem 5.1]) Let p be a prime number, $G$ a finite group of p-power order and $X$ a $\mathbb{Z}_{p}$-homology sphere with $G$-action. Then $X^{G}$ is also a $\mathbb{Z}_{p}$-homology sphere.

Lemma 2.3. (Conner and Floyd [7, Theorem 25.1], cf. [14, Proposition 3.2]) Let $G$ be a finite group of order two and $X$ a connected closed manifold of positive dimension with $G$-action. If $X^{G}$ is a non-empty finite set, then $X^{G}$ consists of an even number of points.

Let $G$ be a finite group and $M$ a manifold with $G$-action. For each $x \in M^{G}$, the tangent space $T_{x}(M)$ of $M$ at $x$ linearly inherits the $G$-action on $M$, i.e. $T_{x}(M)$ is a real $G$-module, and we call the real $G$-module $T_{x}(M)$ the tangential $G$-module at $x$.

The next proposition is a generalization of [15, Proposition 2.9].

Proposition 2.4. Let $G$ be a finite group and $\Sigma$ a homology sphere with $G$-action. Suppose $G$ satisfies the following conditions.

(1) All elements in $G$ have prime power order.

(2) For any $g$ in $G$ such that the order of $g$ is 2-power and greater than four, there is at most one irreducible real $G$-module $V$ with $V^{g}=\{0\}$.

Then the tangential $G$-modules at all points in $\Sigma^{G}$ are mutually isomorphic as real $G$ modules.

Proof. We may assume that $\left|\Sigma^{G}\right| \geq 2$. It suffices to prove that, for any $g \in G$ and for any $x, y \in \Sigma^{G}$, the restrictions to $\langle g\rangle$ of $T_{x}(\Sigma)$ and $T_{y}(\Sigma)$ are isomorphic as real $\langle g\rangle$-modules, where $\langle g\rangle$ denotes the cyclic subgroup of $G$ generated by $g \in G$.

Fix two points $a$ and $b$ in $\Sigma^{G}$. By the hypothesis on the order of elements in $G$, the $g$-fixed point set $\Sigma^{g}$ either is a connected manifold or consists of the two points $\{a, b\}$ for each $g \in G$ (by Smith's theorem). If $\Sigma^{g}$ is connected, then $\operatorname{res}_{\langle g\rangle}^{G} T_{a}(\Sigma)$ is isomorphic to $\operatorname{res}_{\langle g\rangle}^{G} T_{b}(\Sigma)$, and therefore we will verify the isomorphism in the case of $\Sigma^{g}=\{a, b\}$. If the order of $g \in G$ is two, four or an odd-prime power and $\Sigma^{g}=\{a, b\}$, we can obtain $\operatorname{res}_{\langle g\rangle}^{G} T_{a}(\Sigma) \cong \operatorname{res}_{\langle g\rangle}^{G} T_{b}(\Sigma)$ from character theory for $g$ with ord $(g)=2$ or 4 , and from Sanchez' theorem [17, Corollary 1.11] for $g$ with an odd-prime power order. By the condition (2) in Proposition 2.4, for any $g \in G$ with $\operatorname{ord}(g)=2^{r}(r \geq 3)$, both of the tangential $\langle g\rangle$ modules at $\{a, b\}=\Sigma^{g}\left(=\Sigma^{G}\right)$ are generated only by the restriction to $\langle g\rangle$ of one irreducible real $G$-module, and they are thus isomorphic as real $\langle g\rangle$-modules. 
TABLE 3.1. The dimensions of $U^{H}$.

\begin{tabular}{cccccccccc}
\hline & $C_{2}$ & $D_{4}$ & $D_{8}$ & $A_{4}$ & $\mathfrak{A}_{4}$ & $C_{3}^{2} \rtimes C_{2}$ & $S_{4}$ & $\mathfrak{S}_{4}$ & $G$ \\
\hline $\mathbb{R}_{G}$ & 1 & 1 & 1 & 1 & 1 & 1 & 1 & 1 & 1 \\
$U_{5,1}$ & 3 & 2 & 1 & 2 & 0 & 1 & 1 & 0 & 0 \\
$U_{5,2}$ & 3 & 2 & 1 & 0 & 2 & 1 & 0 & 1 & 0 \\
$U_{8, i}(i=1,2)$ & 4 & 2 & 1 & 0 & 0 & 0 & 0 & 0 & 0 \\
$U_{9}$ & 5 & 3 & 2 & 1 & 1 & 1 & 1 & 1 & 0 \\
$U_{10}$ & 4 & 1 & 0 & 1 & 1 & 0 & 0 & 0 & 0 \\
\hline
\end{tabular}

Note that the Mathieu group $M_{10}$ contains an element of order eight, and $A_{6}$ and $M_{10}$ fulfill the conditions (1) and (2) in Proposition 2.4.

Remark 1. As a corollary of Proposition 2.4, for an arbitrary finite group $G$ fulfilling the conditions (1) and (2) in Proposition 2.4, the Smith $\operatorname{set} \operatorname{Sm}(G)$ is trivial. Hence the Smith sets $\operatorname{Sm}\left(A_{6}\right)$ and $\operatorname{Sm}\left(M_{10}\right)$ are trivial, which has already been known by [16, Theorem C3] for $A_{6}$, and by [20, Corollary 4.4] for $M_{10}$.

Proposition 2.5. Let $X$ and $Y$ be closed submanifolds of positive dimensions $m$ and $n$ in $a \mathbb{Z}_{2}$-homology sphere $\Sigma$ of dimension $m+n$ that intersect in all points, transversely. Then the intersection $X \cap Y$ consists of an even number of points.

Proof. Since $\Sigma$ is a $\mathbb{Z}_{2}$-homology sphere of dimension $m+n$, the $\mathbb{Z}_{2}$-intersection form on $\Sigma$,

$$
\varphi: H_{m}\left(\Sigma ; \mathbb{Z}_{2}\right) \times H_{n}\left(\Sigma ; \mathbb{Z}_{2}\right) \rightarrow \mathbb{Z}_{2},
$$

is trivial. This implies that $|X \cap Y| \equiv 0 \bmod 2$.

\section{Actions of $A_{6}$ and $\operatorname{SL}(2,9)$ on $\mathbb{Z}_{2}$-homology spheres}

In this section, we let $G=A_{6}, \widetilde{G}=\operatorname{SL}(2,9)$ and $Z$ be the center of $\widetilde{G}$. Then $G$ and $\widetilde{G}$ have precisely seven and 13 irreducible real $G$ - and $\widetilde{G}$-modules (up to isomorphisms), respectively. We denote the irreducible real $G$-modules by

$$
\mathbb{R}_{G}, U_{5,1}, U_{5,2}, U_{8,1}, U_{8,2}, U_{9} \text { and } U_{10},
$$

where $\operatorname{dim} \mathbb{R}_{G}=1$ and $\operatorname{dim} U_{l}=\operatorname{dim} U_{l, m}=l$, and the irreducible real $\widetilde{G}$-modules by

$$
\mathbb{R}_{\widetilde{G}}, \widetilde{U}_{5,1}, \widetilde{U}_{5,2}, \widetilde{U}_{8,1}, \widetilde{U}_{8,2}, \widetilde{U}_{9}, \widetilde{U}_{10}, W_{8,1}, W_{8,2}, W_{16,1}, W_{16,2}, W_{20,1} \text { and } W_{20,2},
$$

where $\operatorname{dim} \mathbb{R}_{\widetilde{G}}=1$ and $\operatorname{dim} \widetilde{U}_{l}=\operatorname{dim} \widetilde{U}_{l, m}=\operatorname{dim} W_{l, m}=l$. Note that $|Z|=2, \widetilde{G} / Z \cong G$,

$$
\begin{gathered}
\widetilde{U}_{l}^{Z}=\widetilde{U}_{l} \text { and } \quad \widetilde{U}_{l, m}^{Z}=\widetilde{U}_{l, m}, \\
\tilde{U}_{l}^{Z} \cong U_{l} \quad \text { and } \quad \tilde{U}_{l, m}^{Z} \cong U_{l, m} \quad \text { (as real } G \text {-modules), }
\end{gathered}
$$

and that $W_{l, m}^{Z}=0$. Table 3.1 shows $\operatorname{dim} U^{H}$ for some subgroups $H$ of $G$ and for the irreducible real $G$-modules $U$. 
Here, $C_{n}$ and $D_{n}$ denote cyclic and dihedral subgroups of $G$ of order $n$, respectively; $A_{4}$ and $S_{4}$ denote subgroups of $G$ being isomorphic to the alternating and the symmetric groups on four letters, respectively; $\mathfrak{A}_{4}$ and $\mathfrak{S}_{4}$ are subgroups of $G$ that are isomorphic but not conjugate to $A_{4}$ and $S_{4}$ as subgroups of $G$, respectively; and $C_{3}^{2} \rtimes C_{2}$ denotes a subgroup of $G$ being isomorphic to the semidirect product of the elementary abelian group of order nine with a cyclic group of order two acting via the inverse map. Note that $C_{2}, D_{8}$ and $A_{4}$ (respectively $C_{2}, D_{8}$ and $\mathfrak{A}_{4}$ ) are subgroups of $S_{4}$ (respectively $\mathfrak{S}_{4}$ ), and that $C_{2}$ is a subgroup of both $C_{3}^{2} \rtimes C_{2}$ and $D_{4}$.

LEMMA 3.1. Let $\Sigma$ be a $\mathbb{Z}_{2}$-homology sphere with $G$-action. If the $G$-fixed point set $\Sigma^{G}$ is a non-empty finite set, then the set of all points $x$ in $\Sigma^{G}$ such that the tangential G-module $T_{x}(\Sigma)$ is isomorphic to $U_{5,1}^{a_{1}} \oplus U_{5,2}^{a_{2}} \oplus U_{9}^{b} \oplus U_{10}^{c}$ for non-negative integers $a_{1}, a_{2}, b$ and $c$ consists of an even number of points.

Proof. Let $F$ denote the set of all points $x \in \Sigma^{G}$ such that the tangential $G$-module $T_{x}(\Sigma)$ satisfies $\operatorname{dim} T_{x}(\Sigma)^{D_{8}}=\operatorname{dim} T_{x}(\Sigma)^{S_{4}}+\operatorname{dim} T_{x}(\Sigma)^{\mathfrak{S}_{4}}$. For a point $x$ in $F$ and a subgroup $H$ of $G$, we let $\Sigma_{x}^{H}$ denote the connected component of $\Sigma^{H}$ including $x$, and $\Sigma_{F}^{H}$ denote the union of all connected components $C$ of $\Sigma^{H}$ such that $C \cap F \neq \emptyset$. We note that, since $0<\left|\Sigma^{G}\right|<\infty$ (i.e. $\mathbb{R}_{G} \not \subset T_{x}(\Sigma)$ for each $x \in \Sigma^{G}$ ), $x \in F$ if and only if the tangential $G$ module $T_{x}(\Sigma)$ is isomorphic to $U_{5,1}^{a_{1}} \oplus U_{5,2}^{a_{2}} \oplus U_{9}^{b} \oplus U_{10}^{c}$ for non-negative integers $a_{1}, a_{2}, b$ and $c$ (by Table 3.1). Therefore, we will prove that $F$ consists of an even number of points in the following four cases.

Case 1. $\operatorname{dim} T_{x}(\Sigma)^{D_{8}}=0$ for some $x \in F$.

Case 2. $\operatorname{dim} T_{x}(\Sigma)^{D_{8}}=\operatorname{dim} T_{x}(\Sigma)^{S_{4}}(>0)$ for some $x \in F$.

Case 3. $\operatorname{dim} T_{x}(\Sigma)^{D_{8}}=\operatorname{dim} T_{x}(\Sigma)^{\mathfrak{S}_{4}}(>0)$ for some $x \in F$.

Case 4. $\operatorname{dim} T_{x}(\Sigma)^{D_{8}}=\operatorname{dim} T_{x}(\Sigma)^{S_{4}}(>0)+\operatorname{dim} T_{x}(\Sigma)^{\mathfrak{S}_{4}}(>0)$ for all $x \in F$.

Proof in Case 1. By Smith's theorem, $\Sigma^{D_{8}}$ consists of exactly two points, say $\{x, y\}$. Then $\Sigma_{x}^{A_{4}}$ (respectively $\Sigma_{x}^{\mathfrak{A}_{4}}$ ) is a $c$-dimensional closed manifold with $S_{4} / A_{4}$ (respectively $\mathfrak{S}_{4} / \mathfrak{A}_{4}$ )-action, and hence the $S_{4} / A_{4}$ (respectively $\mathfrak{S}_{4} / \mathfrak{A}_{4}$ )-fixed point set consists of the two points $\{x, y\}$ by Lemma 2.3. Thus, we get

$$
\{x, y\}=\Sigma^{D_{8}}=\Sigma^{S_{4}} \cap \Sigma^{\mathfrak{S}_{4}}=\Sigma^{G}=F,
$$

and the proof in Case 1 is complete.

Proof in Case 2. For $x \in F$ with $\operatorname{dim} T_{x}(\Sigma)^{D_{8}}=\operatorname{dim} T_{x}(\Sigma)^{S_{4}}, \Sigma_{x}^{S_{4}}$ is a connected, closed submanifold of a $\mathbb{Z}_{2}$-homology sphere $\Sigma^{D_{8}}$ with the same dimension as $\Sigma^{D_{8}}$. This means that $\Sigma_{x}^{S_{4}}=\Sigma^{D_{8}}=\Sigma^{S_{4}}$. By Table 3.1, $\Sigma^{S_{4}}=\Sigma^{D_{8}}$ and $\left|\Sigma^{G}\right|<\infty$ imply that, for some $a_{1}>0$ and $c \geq 0, T_{y}(\Sigma) \cong U_{5.1}^{a_{1}} \oplus U_{10}^{c}$ for all $y \in \Sigma^{G}$ (hence $F=\Sigma^{G}$ ). Since $\Sigma^{S_{4}}=\Sigma^{D_{8}} \supset \Sigma^{\mathfrak{S}_{4}}$ and $\Sigma^{S_{4}} \cap \Sigma^{\mathfrak{S}_{4}}=\Sigma^{G}, \Sigma^{G}$ coincides with $\Sigma^{\mathfrak{S}_{4}}$. Therefore, to complete the proof in Case 2, we need to show that $\Sigma^{G}$ or $\Sigma^{\mathfrak{S}_{4}}$ consists of an even number of points.

We first consider the case $c=0$. According to Table 3.1, $\Sigma^{D_{4}}$ and $\Sigma_{F}^{C_{3}^{2} \rtimes C_{2}}$ are closed submanifolds of positive dimensions in a $\mathbb{Z}_{2}$-homology sphere $\Sigma^{C_{2}}$, and intersect in all points, transversely. By Proposition 2.5, the intersection $\Sigma^{D_{4}} \cap \Sigma_{F}^{C_{3}^{2} \rtimes C_{2}}=\Sigma^{G}$ consists of an even number of points.

Next consider the case $c>0$. Then $\Sigma_{F}^{\mathfrak{A}_{4}}$ is a $c$-dimensional closed manifold with $\mathfrak{S}_{4} / \mathfrak{A}_{4}$ action. Therefore, $\left|\Sigma^{\mathfrak{S}_{4}}\right| \equiv 0 \bmod 2$ follows from Lemma 2.3 . 
Proof in Case 3. We can prove the proof in Case 3 just as the proof in Case 2, by replacing $a_{1}, U_{5,1}, S_{4}, \mathfrak{A}_{4}$ and $\mathfrak{S}_{4}$ in the proof in Case 2 with $a_{2}, U_{5,2}, \mathfrak{S}_{4}, A_{4}$ and $S_{4}$, respectively.

Proof in Case 4. First, we show that $\Sigma_{F}^{S_{4}} \cap \Sigma_{F}^{\mathfrak{S}_{4}}=F$. It suffices to prove that $\Sigma_{x}^{S_{4}} \cap \Sigma_{x}^{\mathfrak{S}_{4}} \subset$ $F$ for any $x \in F$. Fix a point $x$ in $F$. Then, the tangential $G$-module $T_{x}(\Sigma)$ is isomorphic to $U_{5,1}^{a_{1}} \oplus U_{5,2}^{a_{2}} \oplus U_{9}^{b} \oplus U_{10}^{c}$ for some $a_{1}, a_{2}, b$ and $c$. Let $n_{1}, n_{2}, n_{3}, n_{4}$ and $n_{5}$ be non-negative integers such that

$$
\begin{array}{rlrl}
\operatorname{dim} \Sigma & =5 a_{1}+5 a_{2}+9 b+10 c & =5 n_{1}+5 n_{2}+8 n_{3}+9 n_{4}+10 n_{5}, \\
\operatorname{dim} \Sigma^{C_{2}} & =3 a_{1}+3 a_{2}+5 b+4 c & & =3 n_{1}+3 n_{2}+4 n_{3}+5 n_{4}+4 n_{5}, \\
\operatorname{dim} \Sigma^{D_{8}} & =a_{1}+a_{2}+2 b & & =n_{1}+n_{2}+n_{3}+2 n_{4}, \\
\operatorname{dim} \Sigma_{x}^{S_{4}} & =a_{1}+b & & =n_{1}+n_{4}, \\
\operatorname{dim} \Sigma_{x}^{\mathfrak{S}_{4}} & =a_{2}+b & & =n_{2}+n_{4} .
\end{array}
$$

Since $\Sigma, \Sigma^{C_{2}}, \Sigma^{D_{8}}, \Sigma_{x}^{S_{4}}$ and $\Sigma_{x}^{\mathfrak{S}_{4}}$ are connected, and the determinant of the matrix

$$
\left[\begin{array}{ccccc}
5 & 5 & 8 & 9 & 10 \\
3 & 3 & 4 & 5 & 4 \\
1 & 1 & 1 & 2 & 0 \\
1 & 0 & 0 & 1 & 0 \\
0 & 1 & 0 & 1 & 0
\end{array}\right]
$$

is non-zero (i.e. $n_{1}, n_{2}, n_{3}, n_{4}$ and $n_{5}$ are uniquely determined), the tangential $G$-module $T_{y}(\Sigma)$ is isomorphic to $U_{5,1}^{a_{1}} \oplus U_{5,2}^{a_{2}} \oplus U_{9}^{b} \oplus U_{10}^{c}$ for any $y \in \Sigma_{x}^{S_{4}} \cap \Sigma_{x}^{\mathfrak{S}_{4}}\left(\subset \Sigma^{G}\right)$. This implies that $\Sigma_{x}^{S_{4}} \cap \Sigma_{x}^{\mathfrak{S}_{4}} \subset F$.

Now, by the condition in Case $4, \Sigma_{F}^{S_{4}}$ and $\Sigma_{F}^{\mathfrak{S}_{4}}$ are closed submanifolds of a $\mathbb{Z}_{2}$ homology sphere $\Sigma^{D_{8}}$ intersecting in all points, transversely. Therefore, $F$ consists of an even number of points (by Proposition 2.5).

COROLlary 3.2. Let $\Sigma$ be a $\mathbb{Z}_{2}$-homology sphere with $G$-action. If the $G$-fixed point set $\Sigma^{G}$ consists of an odd number of points, then the set of all points $x$ in $\Sigma^{G}$ such that the tangential $G$-module $T_{x}(\Sigma)$ contains some irreducible real $G$-submodule of dimension eight consists of an odd number of points.

Proof. Lemma 3.1 also says that if $\Sigma^{G}$ is a finite set, the set of all points $x \in \Sigma^{G}$ with the tangential $G$-module $T_{x}(\Sigma)$ not containing any irreducible real $G$-submodule of dimension eight consists of an even number of points. This proves Corollary 3.2.

The next corollary follows directly from Proposition 2.4 and Corollary 3.2.

COROLlary 3.3. Let $\Sigma$ be a homology sphere with $G$-action. If the $G$-fixed point set $\Sigma^{G}$ consists of an odd number of points, then the tangential $G$-module $T_{x}(\Sigma)$ contains some irreducible real $G$-submodule of dimension eight for each point $x$ in $\Sigma^{G}$.

THEOREM 3.4. Let $\Sigma$ be a $\mathbb{Z}_{2}$-homology sphere. If the dimension of $\Sigma$ lies in $T_{G}=$ $[0 . .7] \cup[9 . .12] \cup\{14,15\} \cup\{19,20\}$, then $\Sigma$ does not admit an odd-fixed-point action of $G$. 
Proof. Let $n$ be the dimension of $\Sigma$, and $a, b, c$ and $d$ non-negative integers such that $n=$ $5 a+8 b+9 c+10 d$. Note that $T_{G}$ coincides with the set of non-negative integers defined by

$$
\mathbb{Z}_{\geq 0} \backslash\left\{5 k+8 l+9 m+10 n \mid(k, l, m, n) \in \mathbb{Z}_{\geq 0} \times \mathbb{N} \times \mathbb{Z}_{\geq 0} \times \mathbb{Z}_{\geq 0}\right\},
$$

and if $n \in T_{G}$ then $b=0$. Thus, by Corollary $3.2, \Sigma^{G}$ does not consist of an odd number of points if $n \in T_{G}$.

Next we will discuss odd-fixed-point actions of $\widetilde{G}$ on $\mathbb{Z}_{2}$-homology spheres.

We can obtain the following proposition from Corollary 3.2.

Proposition 3.5. Let $\Sigma$ be a $\mathbb{Z}_{2}$-homology sphere with $\widetilde{G}$-action. If the $\widetilde{G}$-fixed point set $\Sigma^{\widetilde{G}}$ consists of an odd number of points, then the set of all points $x$ in $\Sigma^{\widetilde{G}}$ such that the tangential $\widetilde{G}$-module $T_{x}(\Sigma)$ contains a real $\widetilde{G}$-submodule being isomorphic to $\widetilde{U}_{8,1}$ or $\widetilde{U}_{8,2}$ consists of an odd number of points.

Proof. First, we recall that $|Z|=2, \widetilde{G} / Z \cong G, \widetilde{U}_{8, i}^{Z}=\widetilde{U}_{8, i}$ and $\widetilde{U}_{8, i}^{Z} \cong U_{8, i}$ for $i=1,2$. By Smith's theorem, the $Z$-fixed point set $\Sigma^{Z}$ is a $\mathbb{Z}_{2}$-homology sphere with $G$-action.

Suppose that $\left|\left(\Sigma^{Z}\right)^{G}\right| \equiv 1 \bmod 2$. Then, by Corollary 3.2, there are an odd number of points $x \in\left(\Sigma^{Z}\right)^{G}$ such that the tangential $G$-module $T_{x}\left(\Sigma^{Z}\right)$ contains a real $G$-submodule being isomorphic to $U_{8.1}$ or $U_{8.2}$. Since $\left(\Sigma^{Z}\right)^{G}=\Sigma^{\widetilde{G}}$ and $T_{y}\left(\Sigma^{Z}\right)$ is isomorphic to $T_{y}(\Sigma)^{Z}$ as real $G$-modules for all $y \in \Sigma^{\widetilde{G}}$, we see that for the odd number of points $x \in \Sigma^{\widetilde{G}}$, the tangential $\widetilde{G}$-module $T_{x}(\Sigma)$ contains a real $\widetilde{G}$-submodule being isomorphic to $\widetilde{U}_{8.1}$ or $\widetilde{U}_{8.2}$.

Let $\Sigma$ be a $\mathbb{Z}_{2}$-homology sphere with $\widetilde{G}$-action. An action $\Phi: \widetilde{G} \rightarrow \operatorname{Diff}(\Sigma)$, where $\operatorname{Diff}(\Sigma)$ is the diffeomorphism group of $\Sigma$, is called effective if the kernel

$$
\operatorname{ker} \Phi=\{g \in \widetilde{G} \mid \Phi(g)(x)=x \text { for all } x \in \Sigma\}
$$

of this group homomorphism $\Phi$ is trivial. Hence if an action of $\widetilde{G}$ on $\Sigma$ is effective, the tangential $\widetilde{G}$-module $T_{x}(\Sigma)$ at each point $x \in \Sigma^{\widetilde{G}}$ must contain a real $\widetilde{G}$-submodule being isomorphic to $W_{8,1}, W_{8,2}, W_{16,1}, W_{16,2}, W_{20,1}$ or $W_{20,2}$.

THEOREM 3.6. Let $\Sigma$ be a $\mathbb{Z}_{2}$-homology sphere with effective $\widetilde{G}$-action. If the dimension of $\Sigma$ lies in $T_{\widetilde{G}}=[0 . .15] \cup[17 . .20] \cup\{22,23\} \cup\{27\}$, then $\Sigma$ does not admit an odd-fixed-point action of $\widetilde{G}$.

Proof. By Proposition 3.5, if the $\widetilde{G}$-fixed point set of the effective action of $\widetilde{G}$ on $\Sigma$ consists of an odd number of points, then there are an odd number of points $x \in \Sigma^{\widetilde{G}}$ such that the tangential $\widetilde{G}$-module $T_{x}(\Sigma)$ contains a real $\widetilde{G}$-submodule being isomorphic to $\widetilde{U}_{8.1} \oplus W$ or $\widetilde{U}_{8.2} \oplus W$, where $W$ is one of $W_{8,1}, W_{8,2}, W_{16,1}, W_{16,2}, W_{20,1}$ and $W_{20,2}$.

Let $n$ be the dimension of $\Sigma$, and $a, b, c, d, x, y$ and $z$ non-negative integers such that $n=5 a+8 b+9 c+10 d+8 x+16 y+20 z$. Note that $T_{\widetilde{G}}$ coincides with the set of nonnegative integers defined by

$$
\mathbb{Z}_{\geq 0} \backslash \bigcup_{\alpha=8,16,20}\left\{5 i+8 j+9 k+10 l+\alpha m \mid(i, j, k, l, m) \in \mathbb{Z}_{\geq 0} \times \mathbb{N} \times \mathbb{Z}_{\geq 0} \times \mathbb{Z}_{\geq 0} \times \mathbb{N}\right\},
$$

and if $n \in T_{\widetilde{G}}$ then $b=0$ or $x=y=z=0$. This implies that there is no effective odd-fixedpoint action of $\widetilde{G}$ on $\Sigma$ if $n \in T_{\widetilde{G}}$. 


\section{Actions of $S_{6}, \operatorname{PGL}(2.9), M_{10}$ and $\operatorname{Aut}\left(A_{6}\right)$ on homology spheres}

In this section, we will prove Theorem 1.2, and let $L=A_{6}, G_{1}=S_{6}, G_{2}=\operatorname{PGL}(2,9)$, $G_{3}=M_{10}$ and $G_{4}=\operatorname{Aut}\left(A_{6}\right)$. Each of $G_{1}, G_{2}$ and $G_{3}$ has a subgroup of index two being isomorphic to $L$, and $G_{4}$ has subgroups of index two being isomorphic to $G_{1}, G_{2}$ and $G_{3}$ (hence $G_{4}$ has a subgroup of index four being isomorphic to $L$ ). Thus we may assume that $G_{1}, G_{2}, G_{3}$ and $G_{4}$ have $L$ as a subgroup.

Proposition 4.1. Let $G$ be a finite group, $H$ a subgroup of $G$ with index two and $\Sigma$ a homology sphere with $G$-action. Suppose that either $\Sigma^{H}$ is a finite set or each connected component of $\Sigma^{H}$ has positive dimension. Then $\left|\Sigma^{G}\right| \equiv 1 \bmod 2$ if and only if $\left|\Sigma^{H}\right| \equiv$ $1 \bmod 2$.

Proof. By Proposition 2.1, if $\left|\Sigma^{H}\right| \equiv 1 \bmod 2$ then $\left|\Sigma^{G}\right| \equiv 1 \bmod 2$.

Next assume that $\left|\Sigma^{H}\right| \not \equiv 1 \bmod 2$. By the hypothesis on $\Sigma^{H}, \Sigma^{H}$ either consists of an even number of points or is a closed manifold with $G / H$-action such that each connected component has positive dimension. Therefore, in either case of $\Sigma^{H},\left|\Sigma^{G}\right| \not \equiv 1 \bmod 2$ follows from Proposition 2.1 and Lemma 2.3.

We recall that $L$ and $G_{3}$ fulfill the conditions (1) and (2) in Proposition 2.4. In other words, for $H=L$ or $G_{3}$ and for a homology sphere $\Sigma$ with $H$-action, the tangential $H$ modules at all points in $\Sigma^{H}$ are mutually isomorphic. So the dimensions of all connected components of $\Sigma^{H}$ are equal (i.e. $0<\left|\Sigma^{H}\right|<\infty$ or $\operatorname{dim} \Sigma^{H}>0$ ).

COROllary 4.2. Let $G$ be one of $G_{1}, G_{2}, G_{3}$ and $G_{4}$, and $\Sigma$ a homology sphere with $G$-action. Then $\left|\Sigma^{G}\right| \equiv 1 \bmod 2$ if and only if $\left|\Sigma^{L}\right| \equiv 1 \bmod 2$.

Proof. Corollary 4.2 is obtained by applying Proposition 4.1 once or twice.

Let $G$ be one of $G_{1}, G_{2}, G_{3}$ and $G_{4}$, and $\mathbb{R}_{L}, U_{5,1}, U_{5,2}, U_{8,1}, U_{8,2}, U_{9}$ and $U_{10}$ the irreducible real $L$-modules (up to isomorphisms). The irreducible real $G$-modules $V_{*}$ (up to isomorphisms) and the restrictions to $L$ of $V_{*}$ are as Tables 4.1 to 4.4 .

Here, the real irreducible $G$ - and $L$-modules in Tables $4.1-4.4$ have the following dimensions

$$
\operatorname{dim} \mathbb{R}_{L}=\operatorname{dim} \mathbb{R}_{1, m}=1, \quad \operatorname{dim} U_{l}=\operatorname{dim} U_{l, m}=\operatorname{dim} V_{l}=\operatorname{dim} V_{l, m}=l .
$$

We note that $T_{G_{1}}, T_{G_{2}}, T_{G_{3}}$ and $T_{G_{4}}$ in Theorem 1.2 are alternatively given by

$$
\begin{aligned}
T_{G_{1}} & =[0 . .15] \cup[17 . .20] \cup[22 . .24] \cup[27 . .29] \cup\{33\} \cup\{38\} \\
& =\mathbb{Z}_{\geq 0} \backslash\left\{5 a+9 b+10 c+16 d \mid(a, b, c, d) \in \mathbb{Z}_{\geq 0} \times \mathbb{Z}_{\geq 0} \times \mathbb{Z}_{\geq 0} \times \mathbb{N}\right\}, \\
T_{G_{2}} & =[0 . .7] \cup[9 . .15] \cup[19 . .23] \cup[29 . .31] \cup\{39\} \\
& =\mathbb{Z}_{\geq 0} \backslash\left\{8 a+9 b+10 c \mid(a, b, c) \in \mathbb{N} \times \mathbb{Z}_{\geq 0} \times \mathbb{Z}_{\geq 0}\right\}, \\
T_{G_{3}} & =[0 . .15] \cup[17 . .24] \cup[27 . .31] \cup\{33\} \cup[37 . .40] \cup\{47\} \cup\{49\} \\
& =\mathbb{Z}_{\geq 0} \backslash\left\{9 a+10 b+16 c+20 d \mid(a, b, c, d) \in \mathbb{Z}_{\geq 0} \times \mathbb{Z}_{\geq 0} \times \mathbb{N} \times \mathbb{Z}_{\geq 0}\right\}, \\
T_{G_{4}} & =[0 . .15] \cup[17 . .24] \cup[27 . .31] \cup\{33\} \cup[37 . .40] \cup\{47\} \cup\{49\} \\
& =\mathbb{Z}_{\geq 0} \backslash\left\{9 a+10 b+16 c+20 d \mid(a, b, c, d) \in \mathbb{Z}_{\geq 0} \times \mathbb{Z}_{\geq 0} \times \mathbb{N} \times \mathbb{Z}_{\geq 0}\right\} .
\end{aligned}
$$


TABLE 4.1. Case of $G=G_{1}$.

\begin{tabular}{ccccccc}
\hline$V_{*}$ & $\mathbb{R}_{1, i(i=1,2)}$ & $V_{5, i(i=1,2)}$ & $V_{5, j(j=3,4)}$ & $V_{9, i(i=1,2)}$ & $V_{10, i(i=1,2)}$ & $V_{16}$ \\
$\operatorname{res}_{L}^{G} V_{*}$ & $\mathbb{R}_{L}$ & $U_{5,1}$ & $U_{5,2}$ & $U_{9}$ & $U_{10}$ & $U_{8,1} \oplus U_{8,2}$ \\
\hline
\end{tabular}

TABLE 4.2. Case of $G=G_{2}$.

\begin{tabular}{ccccccc}
\hline$V_{*}$ & $\mathbb{R}_{1, i(i=1,2)}$ & $V_{8, i(i=1,2)}$ & $V_{8, j(j=3,4)}$ & $V_{9, i}(i=1,2)$ & $V_{10, i(i=1,2)}$ & $V_{10,3}$ \\
$\operatorname{res}_{L}^{G} V_{*}$ & $\mathbb{R}_{L}$ & $U_{8,1}$ & $U_{8,2}$ & $U_{9}$ & $U_{10}$ & $U_{5,1} \oplus U_{5,2}$ \\
\hline
\end{tabular}

TABLE 4.3. Case of $G=G_{3}$.

\begin{tabular}{cccccc}
\hline$V_{*}$ & $\mathbb{R}_{1, i(i=1,2)}$ & $V_{9, i}(i=1,2)$ & $V_{10}$ & $V_{16}$ & $V_{20, i(i=1,2)}$ \\
$\operatorname{res}_{L}^{G} V_{*}$ & $\mathbb{R}_{L}$ & $U_{9}$ & $U_{5,1} \oplus U_{5,2}$ & $U_{8,1} \oplus U_{8,2}$ & $U_{10}^{\oplus 2}$ \\
\hline
\end{tabular}

TABLE 4.4. Case of $G=G_{4}$.

\begin{tabular}{cccccc}
\hline$V_{*}$ & $\mathbb{R}_{1, k(k=1,2,3,4)}$ & $V_{9, k(k=1,2,3,4)}$ & $V_{10, i(i=1,2)}$ & $V_{16, i}(i=1,2)$ & $V_{20}$ \\
$\operatorname{res}_{L}^{G} V_{*}$ & $\mathbb{R}_{L}$ & $U_{9}$ & $U_{5,1} \oplus U_{5,2}$ & $U_{8,1} \oplus U_{8,2}$ & $U_{10}^{\oplus 2}$ \\
\hline
\end{tabular}

THEOREM 4.3. Let $G$ be one of $G_{1}, G_{2}, G_{3}$ and $G_{4}$, and $\Sigma$ a homology sphere with $G$ action. If the dimension of $\Sigma$ lies in $T_{G}$, then the $G$-fixed point set $\Sigma^{G}$ does not consist of an odd number of points.

Proof. By Corollary 4.2, it suffices to prove that if $\operatorname{dim} \Sigma \in T_{G}$ then $\Sigma^{L} \not \equiv 1 \bmod 2$.

Suppose that $\operatorname{dim} \Sigma \in T_{G}$ and $0<\left|\Sigma^{L}\right|<\infty$. Then, by the definitions of $T_{G_{1}}, T_{G_{2}}, T_{G_{3}}$ and $T_{G_{4}}$ and by Tables 4.1-4.4, the restriction to $L$ of the tangential $G$-module $T_{x}(\Sigma)$ does not contain any irreducible real $L$-submodule of dimension eight for each $x$ in $\Sigma^{G}$. Thus $\Sigma^{L}$ does not consist of an odd number of points by Corollary 3.3.

Acknowledgements. I would like to thank the referee for pointing out errors and for giving valuable comments. And I am grateful to Professor Masaharu Morimoto for his helpful suggestions and guidance during the course of my studies.

\section{REFERENCES}

[1] A. Bak and M. Morimoto. Equivariant surgery and applications. Proc. Conf. on Topology in Hawaii 1990. Ed. K. H. Dovermann. World Scientific, Singapore, 1992, pp. 13-25. 
[2] A. Bak and M. Morimoto. The dimension of spheres with smooth one fixed point actions. Forum Math. 17 (2005), 199-216.

[3] A. Borowiecka. $S L(2,5)$ has no smooth effective one-fixed-point action on $S^{8}$. Bull. Pol. Acad. Sci. Math. 64 (2016), 85-94.

[4] A. Borowiecka and P. Mizerka. Nonexistence of smooth effective one fixed point actions of finite Oliver groups on low-dimensional spheres. Bull. Pol. Acad. Sci. Math. 68 (2018), 167-177.

[5] G. E. Bredon. Introduction to Compact Transformation Groups. Academic Press, New York, 1972.

[6] N. P. Buchdahl, S. Kwasik and R. Schultz. One fixed point action on low-dimensional spheres. Invent. Math. 102 (1990), 633-662.

[7] P. E. Conner and E. E. Floyd. Differentiable Periodic Maps. Springer, Berlin, 1964.

[8] S. Demichelis. The fixed point set of a finite group action on a homology four sphere. Enseign. Math. 35 (1989), 107-116.

[9] M. Furuta. A remark on a fixed point of finite group actions on $S^{4}$. Topology 28 (1989), 35-38.

[10] E. Laitinen and M. Morimoto. Finite groups with smooth one fixed point actions on spheres. Forum Math. 10 (1998), 479-520.

[11] M. Morimoto. On one fixed point actions on spheres. Proc. Japan Acad. Ser. A Math. Sci. 63 (1987), 95-97.

[12] M. Morimoto. Most of the standard spheres have one fixed point actions of $A_{5}$. Transformation Groups (Lecture Notes in Mathmatics, 1375). Ed. K. Kawakubo. Springer, Berlin, 1989, pp. 240-259.

[13] M. Morimoto. Most of the standard spheres have one fixed point actions of $A_{5}$. II. $K$-Theory 4 (1991), 289-302.

[14] M. Morimoto. A necessary condition for the Smith equivalence of $G$-modules and its sufficiency. Math. Slovaca 66(4) (2016), 979-998.

[15] M. Morimoto and S. Tamura. Spheres not admitting smooth odd-fixed-point actions of $S_{5}$ and $S L(2,5)$. Osaka J. Math. 57 (2020), 1-8.

[16] K. Pawałowski and R. Solomon. Smith equivalence and finite Oliver groups with Laitinen number 0 or 1. Algebr. Geom. Topol. 2 (2002), 843-895.

[17] C. U. Sanchez. Actions of groups of odd order on compact, orientable manifolds. Proc. Amer. Math. Soc. 54 (1976), 445-448.

[18] P. A. Smith. Transformations of finite period. Ann. of Math. 39 (1938), 127-164.

[19] E. Stein. Surgery on products with finite fundamental group. Topology 16 (1977), 473-493.

[20] T. Sumi. Smith set for a simple group. RIMS Kokyuroku 1732 (2011), 101-106.

\author{
Shunsuke Tamura \\ Graduate School of Natural Science and Technology \\ Okayama University \\ 3-1-1 Tsushimanaka \\ Okayama $700-8530$ \\ Japan \\ (E-mail: pvc16v3h@s.okayama-u.ac.jp)
}

